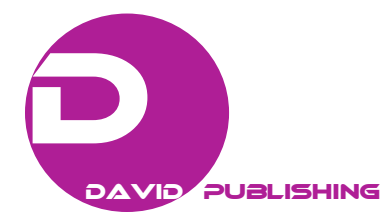

\title{
Polymetrical Analysis as Universal System of Formalization the Knowledge
}

\author{
Petro P. Trokhimchuck \\ Department of Theoretical and Mathematical Physics of Lesya Ukrayinka Eastern European National University, Voly av., 13, Lutsk \\ 43025, Ukraine
}

\begin{abstract}
Problem of search of universal system of formalization the knowledge is discussed. Philisophical, mathematical, cybernetic and computing aspects of its problem are analyzed. Foundation of polymetrical analysis as variant of resolution of this problem is represented. We show that this system allowed resolving S. Beer centurial problem in cybernetics. Also it allowed creating the natural (operational) approach in the foundation of mathematics and system concept of computing science.
\end{abstract}

Key words: Polymetrical analysis, S. Beer centurial problem, triple optimization, hybrid theory of systems, theory of information calculations, foundation of mathematics, variable hierarchy, metascience.

\section{Introduction}

Search of universal system of knowledge has long history [1-4]. The problem of the birth and development of modern science in the context of the development of human civilization is inextricably linked with the emergence and development of the entire human culture [1-4]. Therefore, this problem needs to be considered from this point of view.

All prehistoric cultural and mythological systems can roughly be divided into two types [1,4]. The first relates to ritual systems of the open type: the civilization of the Mesopotamia of the Tigris and the Euphrates, starting from Sumerian and ending with ancient Babylon. The second one is related to ritual systems of the closed (esoteric) type: Ancient Egypt (the cults of the god Thot and the gods of the Sun) $[1,2]$ and the related civilizations of Mexico, Peru and Ecuador [1, 2].

So, let us try to answer the question of how to best describe all the knowledge that was known to different civilizations. We are interested in a brief deductive scheme of this knowledge. English orientalist J. Thomson $[1,2]$ believes that the idea of unity and

Corresponding author: Petro Trokhimchuck, Ph.D., assoc. prof., research fields: msathematics and system analysis. interaction of opposites was the main thing from the very beginning of civilization. It passes through all doctrines - from religion to dual philosophy.

The problem of formalizing knowledge and creating knowledge systems that have developed into modern science has been developed in the era of antiquity. The beginning of this era was begun by Thales and Pythagoras (VII-VI centuries BC), and eventually the closure of the Alexandrian school with the death of Cleopatra $[1,2]$ is sometimes attributed to the time of the life of the Andrianople bishop Yamvlich (V century). The very period of the existence of the Alexandrian school, was begun by the disciple of Aristotle and friend of Makedonian Alexander Ptolemy, the founder of the Ptolemy dynasty in Alexandria after the death of Makedonian Alexander (IV century BC) in Alexandria, and continued to exist sometime until the third and fourth centuries [1-3].

In antiquity three concepts may be selected [1-3].

First is Pythagorean system. Pythagor was synthesized two mythological systems: esoteric Egyptian and open Babilonian-Sumerian.

Second is Aristotle system. He created first classification of science, which has been a basis for modern science. 
Third is Euclidean system. His mathematical classification is basic of modern mathematics.

Later the search of basic ways of creating the science by two, are: inductive, basically experimental science, and deductive, basically theoretical science,

The theoretical sciences were developed in two ways. First, is based on Descartian method [5] and Newtonian four rules of conclusions in physics [6]. Second may be represented as development of Leubniz idea of searching the universal calculation.

First way is caused of creation and developed of modern theoretical physics and other natural theoretical sciences. Basic value of Newtonian method is his four rules:

Rule 1. It is not necessary to require from nature other causes beyond those which are true and sufficient to explain phenomena.

Rule 2. Therefore, as far as possible, we must attribute the same causes to the manifestations of the nature of the same species.

Rule 3. Such properties of bodies that can neither be amplified nor weakenable and which are in all bodies over which tests can be performed must be considered to be the properties of all bodies in general.

Rule 4. In the experimental philosophy, propositions derived from phenomena by means of general induction should be considered to be accurate or approximately correct, despite the possibility of opposing hypotheses, until there are phenomena which they will either be more precise or will be recognized as invalid.

These rules are basic for the creation of basic chapters of modern science. Newtonian laws of mechanics are completion of Descartian synthesis mechanics and mathematics with system point of view $[1,2]$.

Second way is caused by the creation mathematical logic, logical foundation of mathematics and Kleene metamathematics [7]. Kleene added and developed mathematical logic by calculations [8]. Mathematical logic is one of basic chapters of theoretical computer science [9]. The way of development of mathematical logic is next. G. W. Leubniz [10] introduced a law of sufficient satisfication. Boole, Morgan, Peano, Russel and Whitehead created mathematical logic: Boolean algebra and Russel types [10, 11]. Kleene and Wesley [8] created the recursive calculations, which is development of Russel types.

The program of foundation of mathematics must include basic elements of its nature: analysis, synthesis and formalization [11-19]. Logical concept of foundation of mathematics is not corresponding to this condition. Therefore A. N. Whitehead [20] abandoned from his logical concept and said that it must be organism conception. Roughly speaking it must be system concept.

From system point of view Newtonian line is fuller as Leubnizian [1, 2, 21-26]. Real computing operations are based on computer arithmetic. Therefore we must search other system, which can be metamathematics and metascience.

The basic conditions of this metascience must be next:

(1) It must be open theory or theory with variable hierarchy.

(2) This theory must be having minimal number of principles.

(3) It must be based on nature of mathematics (analysis, synthesis and formalization all possible knowledge).

(4) We must create sign structure, which unites verbal and nonverbal knowledge (mathematical and other) in one system.

(5) We must have system, which is expert system of existing system of knowledge and may be useful for the creation of new systems of knowledge.

(6) Principle of continuity must be true for all science.

These conditions must be used for the creation of any dynamic science.

An example of so metascience is Polymetrical Analysis (PA) - theory of variable hierarchy (measure) 
$[1,2]$, which is created as system of optimal analysis, synthesis band formalization of knowledge.

Polymetric analysis (PA) was created as alternative optimal concept to logical, formal and constructive conceptions of modern mathematics and theory of information $[1,2]$.

\section{Basic Concepts of Polymetric Analysis}

Basic concept is formalization of triple minimum: philosophical (methodological), mathematical and concrete scientifically.

Basic elements of this theory and their bonds with other science are represented in Fig. 1 [1, 2].

Firstly we must introduce basic concepts of polymetric analysis, which is necessary for the creation theory of functional-logical automata [1, 2].

Basic mathematical elements of PA are functional elements of quadratic forms (functional numbers) [1, 2].

Second constituents of PA are generalizing mathematical transformations, which are allowing connecting the mathematics (roughly speaking the ordering and formalization) with proper science.

Third constituent of PA is system aspects: creation of proper system of analysis, synthesis and formalization the knowledge.

This concept allows formalizing the Pythagorean phrase "Numbers are ruling the World" [1].

Now we represented basic axiomatic of PA according to Refs. [1, 2, 21-26].

Definition 1. Mathematical construction (constructive) is called set all possible elements, operations and transformations for resolution corresponding problem. The basic functional elements of this construction are called constructive elements.

Definition 2. The mathematical constructive elements $N_{x_{i j}}$ are called the functional parameters

$$
N_{x_{i j}}=x_{i} \cdot \bar{x}_{j},
$$

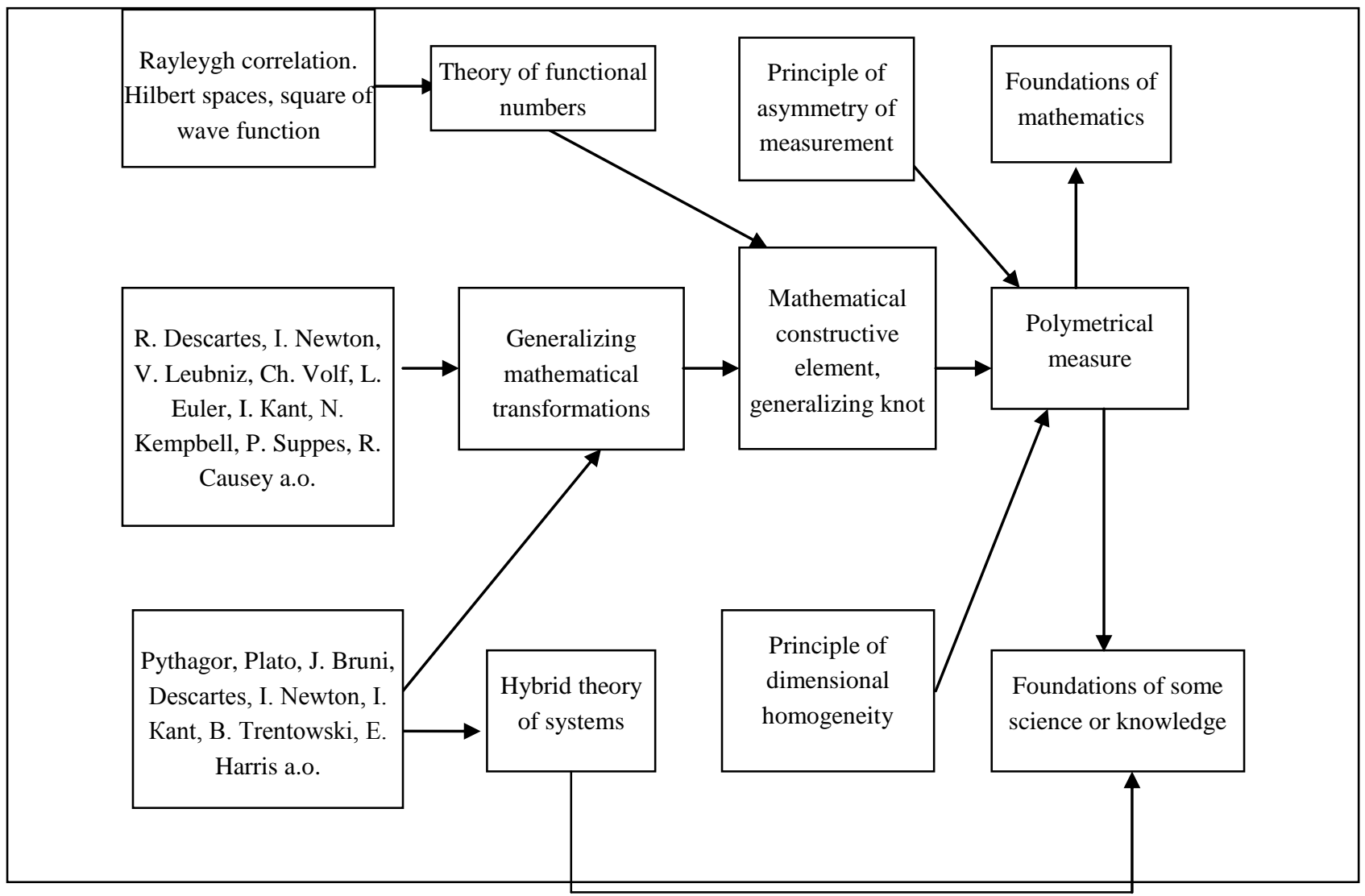


Fig. 1 Scheme of polymetric method and its place in modern science [1, 2].

where $x_{i}, \overline{x_{j}}$ - the straight and opposite parameters, respectively; - - respective mathematical operation.

Definition 3. The mathematical constructive elements $N_{\varphi_{i j}}$ are called the functional numbers:

$$
N_{\varphi_{i j}}=\varphi_{i} \circ \bar{\varphi}_{j}
$$

where

$$
\varphi_{i}\left(x_{1}, \ldots x_{n}, \overline{x_{1}} \ldots \overline{x_{m}}, \ldots N_{x_{i j}}, \ldots\right)
$$

$\overline{\varphi_{j}}\left(x_{1}, \ldots x_{n}, \bar{x}_{1} \ldots \bar{x}_{m}, \ldots N_{x_{i j}}, \ldots\right)$ are the straight and opposite functions, respectively; $\circ$-respective mathematical operation.

Remark 1. Functions $\varphi_{i}, \bar{\varphi}_{j}$ may have different nature: mathematical, linguistic and other.

The theory of generalizing mathematical transformations is created for works on functional numbers $[1,2]$.

Definition 4. Qualitative transformations on functional numbers $N_{\varphi_{i j}}$ (straight $A_{i}$ and opposite $\bar{A}_{j}$ ) are called the next transformations. The straight qualitative transformations reduced the dimension $N_{\varphi_{i j}}$ on $i$ units for straight parameters, and the opposite qualitative transformations reduced the dimension $N_{\varphi_{i j}}$ on $j$ units for opposite parameters.

Definition 5. Quantitative (calculative) transformations on functional numbers $N_{\varphi_{i j}}$ (straight $O_{k}$ and opposite $\bar{O}_{p}$ ) are called the next transformations. The straight calculative trasformations reduced $N_{\varphi_{i j}}$ or corresponding mathematical constructive element on $k$ units its measure. The opposite quantitative transformations increased $N_{\varphi_{i j}}$ or corresponding mathematical constructive element on $l$ units its measure, i.e.

$$
O_{k} \overline{O_{l}} N_{\varphi_{i j}}=N_{\varphi_{i j}}-k \oplus l
$$

Definition 6. Left and right transformations are called transformations which act on left or right part of functional number respectively.

Definition 7. The maximal possible number corresponding transformations is called the rang of these transformations:

$$
\begin{aligned}
\operatorname{rang}\left(A_{i} \bar{A}_{j} N_{\varphi_{i j}}\right) & =\max (i, j) \\
\operatorname{rang}\left(O_{k} \bar{O}_{p} N_{\varphi_{i j}}\right) & =\max (k, p)
\end{aligned}
$$

Remark 2. The indexes $i, j, k, p$ are called the steps of the corresponding transformations.

These transformations may be having various natures. Roughly speaking it may be mathematical, linguistically and other transformations and its combinations

Only 15 minimal basic types of generalizing mathematical transformations exist [1, 2]. These transformatioms are represented in Table 1.

In Table 1 sigh + (plus) is defined that action of corresponding transformation on $N_{\varphi_{i j}}$ is full or particular; sign_-(minus) is absented.

To further describe the theory of information

\begin{tabular}{|c|c|c|c|c|c|c|c|c|c|c|c|c|c|c|c|}
\hline \multirow{2}{*}{ № } & \multirow{2}{*}{$\begin{array}{l}\text { Tronsfor } \\
\text {-mation }\end{array}$} & \multirow{2}{*}{1} & \multirow{2}{*}{2} & \multirow{2}{*}{3} & \multirow{2}{*}{45} & \multirow{2}{*}{56} & & \multirow{2}{*}{\begin{tabular}{l|l|l}
8 & 9
\end{tabular}} & \multirow{2}{*}{10} & \multirow{2}{*}{11} & \multirow{2}{*}{12} & \multicolumn{3}{|c|}{ Representation } \\
\hline & & & & & & & & & & & & & $\mathrm{S}$ & $\mathrm{O}$ & M \\
\hline 1 & f.s. & + & + & & $+t$ & -1 & + & + & + & + & + & + & + & - & - \\
\hline 2 & f. 0. & + & + & + & $+t$ & + & + & + & + & + & + & + & - & + & - \\
\hline 3 & f. $m$. & + & + & + & f & 7 & + & + & + & + & + & + & - & - & + \\
\hline 4 & 1. f.s. & + & - & + & $-t$ & - & + & - & + & - & + & - & - & + & - \\
\hline 5 & r. f. o. & - & + & - & + & + & & + & - & + & - & + & + & - & - \\
\hline 6 & 1. s. & + & - & + & -1 & - & + & - & + & - & + & - & + & - & - \\
\hline 7 & r. 0. & - & + & - & + & + & - & + & - & + & - & + & - & + & - \\
\hline 8 & m.f.s. & + & - & + & -7 & - & + & -1 & + & - & + & - & - & - & + \\
\hline 9 & m. f. o. & - & + & - & + & + & - & + & & + & - & + & - & - & + \\
\hline 10 & 1.h.s. & + & - & - & -1 & + & + & - & & + & - & + & - & + & - \\
\hline 11 & m.h.s. & + & - & - & + & + & + & - & - & - & - & - & - & - & + \\
\hline 12 & r.s. 0. & - & + & + & & & & +1 & + & - & + & - & + & - & - \\
\hline 13 & m.s. o. & - & + & & . & - & & + & & - & + & - & - & - & + \\
\hline 14 & m.s. & + & - & + & - & - & + & + & & + & + & + & - & - & + \\
\hline 15 & m.o. & - & + & & $+1-$ & $t$ & & & & + & + & + & - & - & + \\
\hline
\end{tabular}
calculations (computing), we need to introduce the notion of information lattice $[1,2]$.

Table 1 The basic types of generalizing mathematical transformations.

$\mathrm{S}$ - straight; O-opposite; $\mathrm{M}$ - o.- -full opposite; f. m. - full mixed; 1. f. s. - left full straight; $r$. f. o.-right full opposite; 1. s.- left straight; r. o.- right opposite; m. f. s.-mixed full straight; m. f. o.-mixed full opposite; 1. h. s.-left half-straight; m. h. s.-mixed half-straight; r. s. o.-right semi-opposite; m. s. o.-mixed semi-opposite; m. s.-mixed straight; m.o.—-mixed opposite; 
$1-A_{i} ; 2-\bar{A}_{j} ; 3-A^{r} ; 4-\bar{A}^{r} ; 5-A^{l} ; 6-\bar{A}^{l} ; 7-O_{k} ; 8-\bar{O}_{p} ;$ $9-O^{r} ; 10-\bar{O}^{r} ; 11-O^{l} ; 12-\bar{O}^{l}$.

Definition 8. A generalized knot or generalizing constructive mathematical element is called a ratio:

$$
B={ }_{n m a b}^{s t q o} B_{i j k p}^{\alpha \beta \gamma \ldots}=A_{i_{1}} \ldots A_{i_{\alpha}} \bar{A}_{j_{1}} \ldots \bar{A}_{j_{\beta}} O_{k_{1}} \ldots O_{k_{\gamma}} \bar{O}_{p_{1}} \ldots N_{\varphi_{i j}}
$$

Definition 9. The constructive mathematical structure, which is created on the relations of type (6) is called the information lattice.

The notions of rangs of proper transformations and hierarchy of knots are represented in Refs. [1, 2].

For calculations next concepts were introduced.

Definition 10. Normal arithmetic is arithmetic over real and complex numbers.

Definition 11. The set of mathematical operations and transformations carried out over functional numbers, we call supermodular (functional) arithmetic.

Definition 12. Under modular arithmetic mean arithmetic, particular operations are carried out by the module of a certain number.

Now we represented the general problem of calculation in modern science according to Refs. [1, 2]. This problem is one of central in modern computing science. Systematization and representation of large volume of the information is connected with various technical and mathematical difficulties. In some chapters of information calculation as self-numeric algorithms the general approaches and criteria of estimations the efficiency are absent.

In this case we used the polymetric doctrine, in detail - the measure of calculation (computation). In addition we introduce the concept of informative calculations.

Generalizing mathematical element may represent universal measure of quantity of information [1, 2].

These elements are created informative lattice $[1,2]$. For this case it was called as knots [1, 2]. Informative lattice is basic array of theory of informative calculations. This theory was constructed analogously to the analytical mechanics $[1,2]$.
Basic elements of this theory are:

Definition 13. Informative calculation (computability) $C$ is called the number of possible mathematical operations, which are required for the resolution of proper problem.

Definition 14. Technical informative calculation (computability) is called value:

$$
C_{t}=C \sum_{i=1}^{n} t_{i}
$$

where $t_{i}$-realization time of proper computation.

Definition 15. Generalizing technical informative calculation (computability) is called the quantity:

$$
C_{t g}=k_{A C} C_{t},
$$

where $k_{a c}-\mathrm{a}$ coefficient of algorithmic complexity $[1,2]$.

Basic principle of this theory is the principle of optimal (minimal) informative calculations [1, 2]: any algebraic, including constructive, informative problem has optimal resolution for minimum informative computability $C$, technical informative computability $C_{t}$ or generalizing technical informative computability $C_{t g}$.

Remark 3. The principle of optimal informative calculations is more general as basic principles of information theory as principle of information coding, determination of optimal algorithms and other.

Remark 4. We have taken into account the constructive, but not functional, minimum.

Remark 5. The principle of optimal informative calculations is analogous to action and entropy (second law of thermodynamics) principles in physics and information theory (Shannon theorem) [1, 2, 27].

Idea of this principle may be explained on the basis de Broglie formula [28]:

$$
S_{a} / \hbar=S_{e} / k_{B}=S_{g}
$$

(equivalence of quantity of ordered and disorder information) $[1,2,21-23,25,26]$, where $S_{a}$-action, $\hbar$-Planck constant, $S_{e}$-entropy and $k_{B}$-Boltzman constant and $S_{g}$ may be represented 
as dimensionless measure of information. Therefore we can go from dimensional quantities (action and entropy) to dimensionless quantity-number of proper mathematical operations.

For classification the computations (calculations) on informative lattices hybrid theory of systems (HTS) was created [1, 2]. This theory allows analyzing proper system with point of view of its complexity,

The basic principles of hybrid theory of systems are next: (1) the criterion of reciprocity; (2) the criterion of simplicity.

The criterion of reciprocity is the principle of the creation the corresponding mathematical constructive system (informative lattice). The criterion of simplicity is the principle for the optimization of this creation.

The basic axiomatic of hybrid theory of systems is represented below.

Definition 16. The set of functional numbers and generalizing transformations together with principles reciprocity and simplicity (informative lattice) is called the hybrid theory of systems (in more narrow sense the criterion of the reciprocity and principle of optimal informative calculations).

Criterion of the reciprocity for corresponding systems signed the conservation in these systems the next categories:

(1) the completeness;

(2) the equilibrium;

(3) the equality of the number epistemological equivalent known and unknown knotions.

Criterion of the simplicity for corresponding systems signed the conservation in these systems the next categories:

(1) the completeness;

(2) the equilibrium;

(3) the principle of the optimal calculative transformations.

Criterion of reciprocity is the principle of creation of proper informative lattice. Basic elements of principle reciprocity are various nuances of completeness. Criterion of the simplicity is the principle of the optimality of this creation.

For more full formalization of the all famous regions of knowledge and science the parameter of connectedness $\sigma_{t}$ was introduced. This parameter meant the number of different bounds, the one element of mathematical construction with other elements of this construction, for example, in classic mathematics $\sigma_{t}=1$, in linguistics and semiotics $\sigma_{t}>1$. The parameter of connectedness is the basic element for synthesis in one system of formalization of the all famous regions of knowledge and science. It is one of the basic elements for creation of the theory of functional logical automata too $[1,2]$. The parameter of connectedness may be function too $[1,2]$.

Basic mathematical element of polymetric analysis is functional number (2) [1, 2]. As in Greece mathematics number is basic elements of its system. For these numbers generalizing mathematical transformations were constructed (See formula (6)). Fifteen (15) minimal types of its transformations existed. Informative lattice is constructed on the basis if functional numbers and generalizing mathematical transformations. Theory of informative calculations is created for this lattice. Basic principle of this theory is the principle of optimal calculations.

HTS theory is based on two criterions: criterion of reciprocity-principle of creation of proper formal system, and criterion of simplicity-principle of optimality of this creation. For "inner" bond of two elements of informative lattice a parameter of connectedness $\sigma_{t}$ was introduced. Principle of optimal informative calculation is included in criterion of simplicity.

Using criteria of reciprocity and simplicity and parameter of connectedness the basic famous parts of knowledge and science may be represented as next 10 types of hybrid systems [1, 2, 21-26]:

(1) The system with conservation of all positions for the criteria of reciprocity and simplicity for all elements of mathematical construction (functional 
numbers $N_{\varphi_{i j}}$ and transformations) is called the simple system.

(2) The system with conservation of the criterion of simplicity only for $N_{\varphi_{i j}}$ is called the parametric simple system.

Remark 6. Further in this classification reminder of criteria of reciprocity and simplicity is absented. It means that these criteria for next types of hybrid systems are true.

(3) The system with conservation the criterion of simplicity only for general mathematical transformations is called functional simple system.

(4) The system with nonconservation of the principle of optimal informative calculation and with $\sigma_{t}=1$ is called the semisimple system.

(5) The system with nonconservation of the principle of optimal informative calculation only for $N_{\varphi_{i j}}$ and with $\sigma_{t}=1$ is called the parametric semisimple system.

(6) The system with nonconservation of the principle of optimal informative calculation only for general mathematical transformations and with $\sigma_{t}=$ 1 is called the functional semisimple system.

(7) The system with nonconservation of the principle of optimal informative calculation and with $\sigma_{t} \neq 1$ is called complicated system.

(8) The system with nonconservation of the principle of optimal informative calculation only for $N_{\varphi_{i j}}$ is called parametric complicated system.

(9) The system with nonconservation the principle of optimal informative calculation only for general mathematical transformations and with $\sigma_{t} \neq 1$ is called functional complicated system.

(10) The system with nonconservation of the criteria of reciprocity and simplicity and with $\sigma_{t} \neq 1$ is called absolute complicated system.

With taking into account 15 basic types of generalized mathematical transformations we have 150 types of hybrid systems; practically 150 types of the formalization and modeling of knowledge and science.

Only first six types of hybrid systems may be considered as mathematical, last four types are not mathematical. Therefore HTS may be describing all possible systems of knowledge. Problem of verbal and nonverbal systems of knowledge is controlled with help of types the mathematical transformations and parameter connectedness $[1,2]$.

\section{Polymetric Analysis and Some Problems of Modern Science}

\subsection{Polymetric Theory of Measure and Measurement}

The PA may be represented as general theory of measure and measurement (theory of variable measure) $[1,2]$. It allows including theory of the procedure of measurement with the help of quantitative and qualitative mathematical transformations. Philosophical aspect of this concept was introduced by N. R. Campbell $[29,30]$. The quantitative transformations corresponded to basic (direct) measurements and qualitative transformations corresponded to derivative measurements including dimensional analysis [1, 2]. Therefore polymetric theory of measure and measurements may be represented as formalization Campbell concept $[1,2]$.

The mathematical theory of the polymetric measure is based on functional numbers, generalized mathematical transformations, and the theory of hybrid systems [1, 2]. The main element of the polymetric measure is a generalized mathematical constructive element (6).

We need (6) to associate with the measurement procedure. To do this, you must first set the system type. For measurements, in the classical sense of the word, in this case, the $N_{\varphi_{j}}$ and quantitative transformations corresponded, because actually they (quantitative transformations) are responsible for the arithmetization of the class of intensities (functional numbers $N_{\varphi_{u}}$ ), which itself, precisely speaking, is a measure. This part corresponds to the theory of classical measure. 
Qualitative transformations correspond: firstly, to choose the dimension of a particular measurement; secondly, for the influence of possible errors and corrections to the measurement results; thirdly, they are elements of modeling, forecasting and evaluation of this or that observation element.

The criterion of reciprocity and the principle of the optimal informative calculation are the criteria for the arrangement of the most advantageous mode for obtaining one or another result. Here, in contrast to the usual measurement, the choice of dimension and optimal correlations between empirical and mathematical parts are taken into account. This approach allows not only measuring, but also predicting them.

Let us consider in more detail what we are introducing qualitative transformations. On the one hand, it is a generalization of operations of integration, differentiation, etc., and on the other hand it is a more powerful apparatus of mathematical transformations.

Let us introduce some more auxiliary concepts $[1$, 2].

Definition 17. Transformations are called symmetrical if the rank of direct and inverse transformations are the same.

From the standpoint of classical theory, the introduction of symmetrical straight and opposite transformations merely means an act of measurement is measured or not. In our case, this is true only for quantitative transformations, and in the case of qualitative transformations, this is not so. The fact is that symmetric qualitative transformations allow you to change the measure without changing its dimension, that is, it allows us to make an isomeasurde spectral scale of the measure.

Qualitative transformation is a transformation without a unit or transformation with unit transformations in rank, i.e.

$$
\begin{gathered}
\operatorname{rang} A_{i} \cdot \overline{A_{i}}=0, \\
\operatorname{rang} A_{i} \cdot \bar{A}_{i \pm 1}=\operatorname{rang} \bar{A}_{i \pm 1} \cdot A_{i}= \pm 1 .
\end{gathered}
$$

This approach, in contrast to the classical hierarchical approach, allows us to constructively consider the heterogeneous conditions of measurement and processing of measurement results.

Let us introduce the theory of systems with the polymetric theory of measure. Purely measurable in the classical sense of the word are just simple systems. In all other types of systems, we can, along with the measurements, receive estimates and forecasts, and in completely complex systems, we can only predict.

The basic principles of the theory of measure and measurements may, in our submission, be formulated as follows.

The principle of asymmetry of measurement is formulated in next way $[1,2]$. When the measurement procedure, including the result, can be expressed in the form (6), then next relations are true.

$$
\begin{aligned}
& |k-p| \geq 1 \\
& |q-o| \geq 1 \\
& |a-b| \geq 1
\end{aligned}
$$

Strictly speaking, it is enough that at least one of the three relations is true.

It is easy to see that the relation (11) is a mathematical expression of the measurement asymmetry principle, since quantitative transformations are transformations that correspond to the additive properties of the measure, that is, they can act as both "weights" and as "scales" and as "calculators".

From the analysis of the dimension by virtue of the above, the mathematical construct can be described not necessarily by equations; therefore the most general result can be formulated in this form.

The principle of dimensional homogeneity is formulated in next way $[1,2]$. When the measurement procedure can be represented through (6), for the correct determination of the dimension of the measured value, it is necessary that the condition is fulfilled: 


$$
\begin{aligned}
{ }_{n m a b}^{s t q o} M_{i j k p}= & \delta_{i j}\left(A_{i}, \bar{A}_{j}\right) \delta_{s t}\left(A_{s}^{r}, \bar{A}_{t}^{r}\right) \delta_{n m}\left(A_{n}^{l}, \bar{A}_{m}^{l}\right) \times \\
& \times O_{k} \overline{O_{p}} O_{q}^{r} \bar{O}_{o}^{r} O_{a}^{l} \bar{O}_{b}^{l} N_{\varphi_{i j}},
\end{aligned}
$$

where

$$
\delta_{i j}\left(A_{i}, \bar{A}_{j}\right) ; \delta_{s t}\left(A_{s}^{r}, \bar{A}_{t}^{r}\right) ; \delta_{n m}\left(A_{n}^{l}, \bar{A}_{m}^{l}\right) \quad \text { - corresponding }
$$

to Kronecker algebraic symbols on the corresponding transformations.

In general, the condition (12) can be represented in a more compact form:

$$
\begin{aligned}
& { }_{n m a b}^{s l q o} M_{i j k p}=\delta_{i+s+n, j+t+m}\left(A_{i}, \bar{A}_{j}, A_{s}^{r}, \bar{A}_{t}^{r}, A_{n}^{l}, \bar{A}_{m}^{l}\right) \times \\
& \quad \times f\left(O_{k}, \bar{O}_{p}, O_{q}^{r}, \bar{O}_{o}^{r}, O_{a}^{l}, \bar{O}_{b}^{l}\right) N_{\varphi_{i j}}
\end{aligned}
$$

where $\delta_{i+s+n, j+t+m}$ - summary algebraic Kronecker symbol.

It is easy to see that the relations (12) and (13) are generalizations of the principle of dimensional homogeneity.

Thus, from the above it follows:

Definition 18. The theory of polymetric measurements is a theory built on a plurality of elements, on which the criteria of reciprocity and simplicity are given, as well as the principle of asymmetry of measurement and the principle of dimensional homogeneity.

It has been shown that the basic theories of measure and measurement are particular cases of polymetric theory of measure and measurement $[1,2]$.

\subsection{Polymetric Analysis and Foundations of Mathematics}

But each science is characterized of set proper concepts, principles and laws. Therefore we must select concept and numbers and types of basic principles and laws for creation new metascience theory, which corresponded to present state of modern science and may be used for its development.

Basic concepts of foundation of mathematics are next [7-18]: logical (B. Russel and A. Whihead; formalistic (D. Hilbertn and P. Bernays) and intuitionistic (L. Brauer and A. Heyting).

First is based on logic, arithmetic or set theory. But these theories are closed theories and are based on procedure of formalization of the knowledge. Therefore in this case the inter-hierarchic paradoxes, as Russel paradox, existed [9, 12]. These two concepts neglect the problem of selection of proper mathematical construction.

Intutionistic (constructive) concept is based on the problem of selection of the proper mathematical construction for the resolution the corresponding problem [9, 12].

Large role in modern science has a problem of completeness in the proper system or theory. In epistemological sense the notion is equivalence for the concept of closing in theory of system $[1,2,26]$.

The concept of completeness is basic in mathematical logic and was developed by K. Gödel.

His two incompleteness theorems may be represented in next forms $[1,9,12,31]$.

First Incompleteness Theorem: That if the arithmetic is consistent, then there exists an irreducible and irrefutable formula in it.

This theorem may be represented in more general form as "Any consistent formal system $F$ within which a certain amount of elementary arithmetic can be carried out is incomplete; i.e., there are statements of the language of $F$ which can neither be proved nor disproved in $F$."

The improvable statement $G_{F}$ referred to the theorem is often referred to as "the Gödel sentence" for the system $F$. The proof constructs a particular Gödel sentence for the system $F$, but there are infinitely many statements in the language of the system that shares the same properties, such as the conjunction of the Gödel sentence and any logically valid sentence.

For each formal system $F$ containing basic arithmetic, it is possible to canonically define a formula $\operatorname{Cons}(F)$ expressing the consistency of $F$. This formula expresses the property that "there does not 
exist a natural number coding a formal derivation within the system $F$ whose conclusion is a syntactic contradiction." The syntactic contradiction is often taken to be " $0=1$ ", in which case $\operatorname{Cons}(F)$ states "there is no natural number that codes a derivation of ' $0=1$ ' from the axioms of $F$."

Gödel's second incompleteness theorem shows that, under general assumptions, this canonical consistency statement $\operatorname{Cons}(F)$ will not be provable in $F$. The theorem first appeared as "Theorem XI" in Gödel's paper [33]. In the following statement, the term "formalized system" also includes an assumption that $F$ is effectively axiomatized theory.

Second Incompleteness Theorem: States that if formal arithmetic is consistent then there is an irreducible formula that substantiates the contradiction of the arithmetic.

This theorem is stronger than the first incompleteness theorem because the statement constructed in the first incompleteness theorem does not directly express the consistency of the system. The proof of the second incompleteness theorem is obtained by formalizing the proof of the first incompleteness theorem within the system $F$ itself.

From system point of view these theorems may be represented as particularly formalization of rule 3 from Newtonian's rules of conclusions in physics.

The problem of completeness may be transited on all closed systems.

Polymetric analysis is optimal synthesis of all three concepts of foundation of mathematics formalization and selection of mathematical construction (or analysis and synthesis).

But basic criteria of PA (reciprocity and simplicity) are based on concept of completeness. In whole HTS is theory of open systems with variable hierarchy, therefore Gödels theorems has not basic value in this theory.

\subsection{Polymetric Analysis as Metascience}

The large value in modern science has concepts of reduction and resolutions of various problems of modern science. Problem of resolution of proper problems may be represented as reduction or transformation for this problem in form, which is necessary for using.

In mathematical physics many complex problems of nonlinear Hamiltonian dynamics were reduced to systems of proper linear equations by famous Japanese mathematician M. Sato with colleagues [1, 2]. Therefore we are formulated general theorem of reduction as general Sato theorem $[1,2]$.

This theorem was expanded on classification and used for the creation of Relaxed Optics [1].

Polymetric analysis may be used for the resolution of many problems of modern science in whole and with using theories. These problems are included in its structure.

PA may be represented as "dynamical" expanding formalization of Errol E. Harris polyphasic concept of modern science [32, 33]. But Harris method is philosophical and "static", polymetric method is "dynamic". PA allows selecting and changing measure and hierarchy of proper systems.

So, HTS may be used for the classification and creation of old and new chapters of all science, including computing science [1, 2, 34-39].

HTS may be used for the represented evolution of systems in two directions: (1) from simple system to complex system (example, from classic to quantum mechanics) and (2) conversely, from complex system to simple system (example, from formal logic to mathematical logic) [1,2].

Hybrid theory of systems is open theory. Parameters of openness are number of generalizing mathematical transformations and parameter of connectedness. Thereby we have finite number of types of systems, but number of systems may be infinite. Hybrid theory of systems allows considering verbal and nonverbal knowledge with one point of view $[1,2]$. Therefore this theory may be represented as variant of resolution S. Beer centurial problem in 
cybernetics (problem of complexity) [34, 36, 37, 39].

HTS may be represented as application PA (theory of informative calculations) to the problem of calculation $[1,2,21]$. This theory was used for the problem of matrix computation and problem of arrays sorting $[1,2,21]$.

HTS may be connected with problem of computational complexity. This problem was appeared in modern cybernetics for resolution of problem of the transition from infinite (analytical) to discrete representation of computing procedures $[1,2$, 21]. It may be connected with 4 and 5 Smale problems $[35,36]$.

HTS may be used for the classification of knowledge and science with point of view of their complexity. These results may be represented as theorems [1, 2].

Theorem 1. The classical mechanics is the simple system.

Proof. The classical mechanics is closed system therefore criteria of the reciprocity and simplicity are true. The action principle is analogous with the principle of optimal informative calculations. Parameter of connectedness is equal to 1 . But its definition of simple system and theorem is proved.

Theorem 2. The quantum mechanics is the semisimple system: (1) in Heisenberg's representation-parametric simple; (2) in Shrödinger's representation - functional simple; (3) in the representation of interaction-semisimple system.

Proof. The quantum mechanics is closed system as classical mechanics. But the criterion of the simplicity is not true for operators (in Heisenberg's representation), for wave functions (in Shrödinger's representation) and for operators and wave functions (in representation of the interaction). Parameter of connectedness is equal to 1 for all three representations. But it is the definitions of proper systems and theorem is proved.

Theorem 3. Logic is a simple system.

Proof. Logic is a closed system. Criteria for reciprocity and simplicity of the system are implemented. Parameter connectivity equaled one. Thus mathematical logic is a simple system.

Theorem 4. Linguistics is a complex system.

Proof. Linguistics is semi-closed or open system. Criteria reciprocity and simplicity may not come true. Parameter connectivity, typically, is greater than one. But it is the definition of a complex system.

These theorems practically represented the system character of the theoretical (classic) and quantum mechanics in modern science.

Once again we return to the foundations of mathematics. Classical mathematics is characterized by parameter of connectedness that is equal to one. It means that quite complex and sophisticated mathematical system is not mathematical in the classical sense. But the foundations of mathematics we have a theory with a broader subject base as classical mathematics (including mathematical logic and set theory). This theory in our view should include formalizing the procedure (functional numbers and criteria of reciprocity and simplicity), process analysis and synthesis (qualitative and quantitative transformation) and the problem of uniqueness (parameter of connectedness). This theory is also essential to have provisions that take into account its opening from the system point of view. Polymetric analysis meets this requirement parameter of connectedness and possible failure of certain provisions of criteria of reciprocity and simplicity. The theories of "structural lines" in the foundations of mathematics do not meet these requirements. This provision can be formulated as the following theorem.

Theorem 5. The theory of "structural lines" in the foundations of mathematics (logical, formal and intuitive) cannot be extended to all mathematics.

Proof. The theory of "structural lines" is permanent measures, while mathematics because of its development has a variable structure, and each structural element has its own measure. But metamathematycal theory must be theory with 
variable measure. We got a contradiction and thus proved the theorem.

If we consider polymetric concept in terms of $\mathrm{H}$. Kantor expression [2, 21] "The essence of mathematics lies in its freedom", this freedom is included in the variable measure.

Polymetric analysis may be represented as generalization of basic problem of cybernetics in Wiener sense "Cybernetics is the science of the Control and Communication in the Animal and the Machine" [40].

But PA is more general as cybernetics. It may be used as metascience and expert system for real systems and theory of formation of new scientific systems $[1,2]$.

PA is universal system of synthesis of knowledge. But this synthesis is realized through measure (number). Each science or knowledge has own treasures and measure. Therefore problem of division science on philosophy and other sciences (see N. R. Campbell $[29,30])$ is very relative. The classification science and knowledge with help polymetric concept, according to simplicity-complexity of optimal formalization, is fuller and more corresponded to present state and development of science and knowledge.

Selection of quadratic forms as basic elements of PA is further development of direction of observation for many scientists: Pythagor (Pythagorean theorem), Plato (three types of numbers), Descartes (using Pythagorean theorems for creation analytical geometry), B. Riemann (creation Euclidean space as generalizing of analytical geometry), D. Hilbert (Hilbertian spaces) etc. [1, 2].

PA may be used as theoretical foundations of computer science too. It describes this science in your standing and development more simple, optimal and sufficient as logical or constructive concepts $[1,2]$, and may be used as expert system for existing sciences and instrument for creation of new sciences.

Polymetric analysis may be used for more full formalization of neuronets $[1,2]$. Basic concept of creation PA is concept of triple optimum (minimum): mathematical, methodological and concrete scientific $[1,2]$, therefore it may be used for all possible systems of knowledge.

The polymetric analysis may be represented as universal theory of synthesis in Descartian sense. For resolution of this problem we must select basic notions and concepts, which corresponded to optimal basic three directions of Fig. 1.

Thus, we show that polymertric analysis is general theory of open systems and may be used for the resolutions of various problems of system type for many sciences. It may be represented as metamathematics is more widely sense as "logical" Kleene metamathematics [7]. PA may be represented as metaknowledge, metascience and metamathematics together in one system.

If seeing development of science with system point of view we have to general classifications. First is Euclid's "Elements" as classification of basic chapters of mathematics [1, 2, 21]. Second is Aristotle's classification of science. Roughly speaking, modern mathematics is the expansion of Euclid's "Elements", and modern science is expansion of Aristotle's classification.

But modern science and mathematics is more complex systems as in Euclides and Aristotle times. It is polyphasic system. But it must be open system. This metascience must include elements of integration (synthesis) and differentiation of knowledge and science. It must be theory with variable measure and hierarchy.

PA may be represented as more general theory of everything as physical theories of everything, including anthropic principle [41-43].

Therefore we must include the elements of simplicity-complexity of these systems for its comparative analysis. S. Beer problem of century in cybernetics is one of central problems of modern knowledge and science, including mathematics (two 
Smale problems) too. This problem has two aspects: first-system (global) and second-local scientifically.

The concept of PA is third way of classification of science. But it is "dynamical" concept, which is based on variable measure, problem of simplicity-complexity and two "open" parameters number and types of generalizing mathematical transformation and parameters of connectedness.

From mathematical point of view PA is expansion of mathematics in all possible systems of knowledge and science with point of view that the simplicity-complexity of mathematics must be no instrument for the resolve of pure mathematical problems. It must be expert system for old systems of knowledge and science and scientific prognostication system for new systems of knowledge and science.

Such expansions of mathematics corresponded to basic thesis of computer science and allow bonding mathematics (précised knowledge), science (ordered knowledge) and other knowledge, including culture and religious, in one system.

PA may be represented as expansion of the Newtonian method (four rules of conclusions in physics) on all science and knowledge. But Newton had problem of ended second Descartian synthesis (classical mechanics) and transit from complex system to more simple [21]. Basic problem of PA is creation of universal system of formalization of knowledge and basically the transit from local (more simple) to global (more complex) problems.

PA may be represented as more full formalization E. B. de Condillac A Treatise about Systems [44]. But in this case we have open systems, systems with variable measure and variable hierarchy. These both conditions are interconnected and therefore it represents the one universal system.

PA may be represented as system in E. B. de Condillac sense about minimal number of basic principles [44]. Only two criteria (reciprocity and simplicity) allow formalizing all knowledge.
If mathematics is précised Knowledge [45] and other science and knowledge are "ordered knowledge" [21] then in polymetric analysis they are systems with various steps of complexity $[1,2,21]$.

PA may be represented as answer to E. Wigner problem of creation of the more universal system science or metascience [21]. He said that necessity in stronger science integration caused the reciprocal of mathematics, physics and psychology for natural sciences. But development of computing science allows transiting this problem on all science and knowledge. Therefore, only generalizing computing science as PA may represent the universal system of foundation of science.

Thus we show that PA is satisfied to the six conditions of creation the metascience, which were formulated in introduction of this paper.

\section{Conclusions}

(1) The problems of creation the universal systems of the knowledge are discussed.

(2) Basic requirements to creation of universal system of formalization of knowledge were formulated.

(3) Basic concepts of Polymetric Analysis as variant the resolution of problem of the optimal formalization of knowledge are represented.

(4) Hybrid theory of systems is represented as universal system of formalization the knowledge.

(5) S. Beer centurial problem in cybernetics and its resolution with help the methods of polymetric analysis is observed.

(6) It was shown that Polymetric Analysis may be represented as natural approach in foundation of mathematics and operational concept in the foundation of computer science.

(7) Polymetric theory of measure and measurements (theory of variable measure) is analyzed as variant of universal theory of measure and measurements.

(8) The problem of synthesis verbal and nonverbal systems of knowledge in one system is discussed too. 


\section{Acknowledgment}

I thank by N. Nepeyvoda, A. Ershov, A. Kifishin, M. Bunge, Yu. Golovin, A. Balandin, A. Kuzmich, N. Engver, V. Tkalych, A. Kuhtenko, S. Nikolskiy, N. Moiseev, A. Sakharov, I. Shafarevich, V. Chabanyuk, Ya. Rudnitskiy, F. Grier, E. Harris and A. Ivakhnenko for discussions of basic concepts and D. Shvalikovskiy and O. Viligurskiy for help in the preparation of this paper.

\section{References}

[1] Trokhimchuck, P. 2014. Mathematical Foundation of Knowledge. Polymetrical Doctrine. Vezha-Print, Lutsk. (in Ukrainian).

[2] Trokhimchuck, P. 2018. Polymetrical Analysis, History, Concepts, Applications. Lambert Academic Publishing.

[3] Russel, B. 1945. A History of Western Philosophy and Its Connection with Political and Social Circumstances from the Earliest Times to the Present Day. Simon and Schuster.

[4] Van der Waerden, B. L. 1985. Science Awakening Science. The Birth of Mathenatics. New York: Oxford University Press.

[5] Fisher, K. Descartes. St. Petersburg: MIFRIL. (in Russian)

[6] Newton, I. 1846. Mathematical Principles of Natural Philosophy. New York: Daniel Adee Published.

[7] Kleene, S. C. 1952. Introduction to Metamathematics. North Holland, Amsterdam.

[8] Kleene, S. C., and Wesley, R. 1965. The Foundations of Intuitionistic Mathematics, Especially in Relation to Recursive Ffunctions. North Holland, Amsterdam.

[9] Nepeyvoda, N. N. 2001. Applied Logic. Novosibirsk. University Press. (in Russian)

[10] Leubniz, G. 1984. "Studies of Universal Calculus." In Works in Four Volumes, Vol. 3, Gottfried Wilhelm Leubniz. Moscow: Mysl', pp. 533-7. (in Russian)

[11] Russel, B. 1948. Introduction to Mathematical Philosophy. London: Museum Street.

[12] Ruzha, I. 1981. Foundations of Mathematics. Kiev: Vyshcha Shkola. (in Russian)

[13] Bogachevskiy, Y. 1937. "Formalism and Intuitionism in Mathematics." Transactions of Shevchenko Scientific Society 31: 81-98. (in Ukrainian)

[14] Kuhtenko, A. 1987. Cybernetics and Fundamental Science. Kyiv: Naukova Dumka. (in Russian)

[15] Hilbert, D., and Bernays, P. 1984. Foundations of Mathematics. Moscow: Mir. (in Russian)

[16] Styazhkin, N. I. 1967. The Formation of Mathematical
Logic. Moscow: Nauka. (in Russian)

[17] Kline, M. 1985. Mathematics and the Search for Knowledge. New York: Oxford University Press.

[18] Kline, M. 1982. Mathematics. The Loss of Certainty. New York: Oxford University Press.

[19] 1987. Fundamental Problems of Modern Mathematics. Moscow: Nauka. (in Russian)

[20] Whitehead, A. 1948. Science and the Modern World. New York: Pelican Mentor Books.

[21] Trokhimchuck, P. 2017. "Effective Computations and Foundations of Science." International Journal on Recent and Innovation Trends in Computing and Communication 5 (10): 162-71.

[22] Trokhimchuck, P. 2016. "Polymetrical Analysis: Retrospective and Perspective." International Journal on Recent and Innovation Trends in Computing and Communication 4 (1): 173-83.

[23] Trokhimchuck, P. 2016. "Problem of Simplicity-Complexity in Modern Science and Polymetrical Analysis." International Journal of Engineering Research and Management 3 (7): 86-95.

[24] Trokhimchuck, P. 1992. "To Question of the Creation of Universal Theory of Systems." Reports of Russian Academia of Science 326 (3): 441-5. (in Russian)

[25] Trokhimchuck, P. 1992. "Synthesis and Unification in the Science, Aspects of the Formalization." Bulletin of Ukrainian Academy of Science 6: 26-33. (in Ukrainian)

[26] Trokhimchuck, P. 2017. "Theories of Open Systems: Realities and Perspectives." International Journal of Innovative Science and Research Technology 2 (4): 51-60.

[27] Brillouin, L. 2004. Science and Information Theory. New York: Courier Corporation.

[28] De Broglie, L. 2014. "Thermodynamics of Isolated Point (Hidden Thermodynamics of Particles)." In Collected Papers, vol. 4, edited by L. de Broglie. Moscow: Print-Atel'e, pp. 8-111. (in Russian)

[29] Campbell, N. R. 1920. Physics: In Elements. Cambridge University Press.

[30] Campbell, N. R. 1928. An Account of the Principles of Measurements and Calculations. London: Longman Green.

[31] Gödel, K. 1992. On Formally Undecidable Propositions in Principia Mathematica and Related Systems I. New York: Dower Publications, Inc.

[32] Harris, E. E. 1965. The Foundations of Metaphysics in Science. London: George Allen Unwin.

[33] Harris, E. E. 1970. Hypothesis and Perception. London and New York: George Allen Unwin Humanities Press.

[34] Beer, S. 1976. "We and Complexity of Modern World." In Cybernetics Today: Problems and Propositions. Moscow: Znaniye, pp. 7-32. (in Russian) 
[35] Smale, S. 1998. "Mathematical Problems for the Next Century." Mathematical Intelligencer 20 (2): 7-15.

[36] Smale's Problems. Wilkipedia-The Free Encyclopedia. http:/en. wikipedia.org/wiki/Smale'sproblems.

[37] Yudin, D. V., and Yudin, A. D. 1985. "Mathematicians Measure Complexity." Number and Thought 8: 192. (in Russian)

[38] Casti, J. 1979. Connectivity, Complexity and Catastrophe in Large-Scale Systems. New York: John Wiley \& Sons.

[39] Uemov, A. I. 1973. Types and Criteria of Simplicity of Systems. Kyiv: Prep. Institute of Cybernetics, 73-9. (in Russian)

[40] Wiener, N. 1983. Cybernetics or Control and
Communication in the Animal and the Machine. Moscow: Nauka. (in Russian)

[41] Barrow, J. D. 1998. Impossibility. The Limits of Science and Science of Limits. Oxford University Press.

[42] Barrow, J. D. 2012. New Theories of Everything: The Quest for Ultimate Explanation. Minsk: Popurry. (in Russian)

[43] Barrow, J. D., and Tippler F. 1986. The Anthropic Cosmological Principle. Oxford University Press.

[44] De Condillac, E. B. 1982. A Treatise about Systems. Lawrence Erlbaum Associates, Inc., pp. 1-174.

[45] Arnold, V. 2002. What Is Mathematics? Moscow: MTNMO. (in Russian) 